\title{
И снова о принципе П. Кюри
}

Войтеховский Ю.Л. 1,2

${ }^{1}$ Санкт-Петербургский горный университет, voytekhovskiy_yul@pers.spmi.ru

${ }^{2}$ Геологический институт КНЦ РАН, Anamumbl,woyt@geoksc.apatity.ru

Аннотация. Статья посвящена рассмотрению новых интерпретаций принципа диссимметрии Кюри в статье Б.С. Левина (2018) и книге В.П. Афанасьева (2018). Показано, что корректное понимание категорий симметрии, диссимметрии и принципа Кюри в целом не нуждается в расчленении восприятия природы на логический и онтологический уровни. Обращено внимание на то, что принцип Кюри относится к суперпозициям однородных явлений. Суперпозиции разнородных явлений следует разделять на однородные части с помощью методов относительной или абсолютной геохронологии. Предложены направления дальнейших исследований принципа Кюри.

Ключевые слова: симметрия, диссимметрия Пастера, диссимметрия Кюри, принцип Кюри, предельные группы симметрии.

\section{Once again about the $P$. Curie principle}

Voytekhovsky Y.L. ${ }^{1,2}$

${ }^{1}$ Saint-Petersburg Mining University, voytekhovskiy_yul@pers.spmi.ru

${ }^{2}$ Geological Institute of KSC RAS, Apatity,woyt@geoksc.apatity.ru

\begin{abstract}
The article is devoted to the consideration of new interpretations of the Curie dissymmetry principle in the book by V.P. Afanasyev (2018) and the article by B.S. Levin (2018). It is shown that correct understanding of the symmetry and dissymmetry categories, and the Curie principle as a whole does not need the nature perception to be divided into the logical and ontological levels. It is noticed that Curie's principle relates to superpositions of homogeneous phenomena. Superpositions of heterogeneous phenomena should be preliminary divided into homogeneous parts using the methods of relative or absolute geochronology. Directions for further research on the Curie principle are suggested.
\end{abstract}

Key words: symmetry, Pasteur's dissymmetry, Curie's dissymmetry, Curie's principle, limit symmetry groups.

\section{Введение}

В Трудах XIII Ферсмановской научной сессии опубликованы статьи Б.С. Левина (2016 а-г), обозначившие редкий ныне интерес к методологическим вопросам геологии. В 2018 г. вышла его очередная статья (Левин, 2018), на этот раз о принципе диссимметрии П. Кюри (Curie, 1894, 1908; Кюри, 1966). Далее мы называем его именно так, поскольку в формулировках самого П. Кюри акцент сделан именно на роли диссимметрии. Следом вышла в свет книга (Афанасьев, 2018), тоже затрагивающая принцип диссимметрии П. Кюри. Тем более интересно появление сразу двух работ, одна из которых тяготеет к минералогии, другая - к геологии. ${ }^{1}$

Теоретическое содержание этого принципа (Шубников, 1946, 1951 а, 1951 6, 1956, 1961, 1966, 1972) и его приложения к геологическим и биологическим системам разного уровня сложности (Шафрановский, 1954, 1956, 1957, 1959, 1964, 1966, 1968 а, 1968 б, 1971 а, 1971 б, 1972, 1974 a, 1974 б, 1975; Шафрановский и др., 1972; Шафрановский, Плотников, 1975) - некогда популярная тема в отечественной и зарубежной литературе (Sander, 1930, 1948, 1950; Paterson, Weiss, 1961; Turner, Weiss, 1963; Kirchmayer, 1965, 1966, 1968; Amstutz, 1966). Затем в её обсуждении имел место долгий перерыв. В относительно недавних статьях (Войтеховский, 2005; Войтеховский, Степенщиков, 2005; Voytekhovsky, Stepenshchikov, 2004; Voytekhovsky, 2005) принцип Кюри использован для объяснения искажённых кристаллов альмандинов Кольского п-ова.

Сам П. Кюри был не вполне доволен формулировкой принципа диссимметрии в основополагающей статье (Curie, 1894, 1908). Об этом свидетельствует М. Кюри (1924). А.В. Шубников

\footnotetext{
${ }^{1}$ Достаточно полный список работ, посвящённых принципу Кюри, дан в конце статьи. - Ю.В.
} 
(1956) отмечает: «Для работ П. Кюри по симметрии, как, впрочем, и для всех его работ, характерна чрезвычайная краткость их изложения. <... Если указанная сжатость изложения нисколько не затрудняет чтения большинства работ П. Кюри, этого нельзя сказать об его исследованиях по симметрии. Возможно, что именно это обстоятельство было причиной того, что они не были достаточно поняты и оценены физиками» (с. 591). И далее: «Идеи П. Кюри в области учения о симметрии нельзя считать до конща оформленными. Это сделают будущие поколения» (с. 602). И.И. Шафрановский $(1964,1966)$ обратился к изучению русского перевода статей П. Кюри с целью возможного «уточнения универсального приничиа симметрии» и подтвердил, что трудности понимания связаны не с якобы недостаточной точностью перевода.

Всё это, очевидно, создаёт предпосылки дискуссии. Чтобы не добавлять к ней лишних поводов, подчеркнём, что далее «диссимметрия» понимается именно по П. Кюри, а не по Л. Пастеру (1937, 1960, Pasteur, 1853, 1861), определившему её (в современной терминологии) как отсутствие у конечных фигур элементов симметрии 2-го рода или, в другой формулировке, как наличие у конечных фигур лишь поворотных осей. Теоретическое обсуждение пастеровского понимания «диссимметрии» тоже не закончено. А его приложения к природным объектам и средам не исчерпываются констатацией преобладающей правизны или левизны у биологических объектов и их паритета в мире минералов, но простираются до анализа структуры вакуума (Шубников, 1961).

О терминологии добавим следующее. «В широких научных кругах диссимметрию - идёт ли речь о диссимметрии Л. Пастера или о диссимметрии П. Кюри - часто смешивают с асимметрией, т.е. полным отсутствием симметрии. Асимметрия, очевидно, является лишь частным случаем диссимметрии (за исключением тривиального случая, когда фигура изначально асимметрична и не может быть более диссимметризована. - Ю.В.). Диссимметрию смешивают иногда и с антисимметрией - противоположной симметрией, описываемой специальными группами четырёхмерной симметрии» (Шубников, 1956, с. 598).

Наконец, имеет хождение термин «дисимметрия», то есть «двойная симметрия». Насколько известно автору, он никем не определялся по существу, не имеет отношения к билатеральной симметрии $(m)$ или любой другой точечной группе симметрии порядка 2 и должен быть устранён из употребления как недоразумение, вносящее путаницу.

\section{Принцип Кюри в работах А.В. Шубникова}

П. Кюри сформулировал несколько согласованных положений, выражающих принцип диссимметрии в совокупности. Все они рассмотрены А.В. Шубниковым (1956). «Характеристическая для того или иного явления симметрия есть максимальная симметрия среды, совместимая с существованием явления. Явление может существовать в среде, которая обладает либо характеристической симметрией, либо одной из её подгрупп. Иначе говоря, некоторые элементы симметрии среды могут сосуществовать с явлением, но они не являются обязательными. Обязательным является лишь отсутствие некоторых элементов симметрии. Это она - диссимметрия - творит явления〉 (с. 596).

А.В. Шубников поясняет это положение эффектным - в прямом и переносном смыслах - примером. Пироэффект возможен лишь в средах с характеристической симметрией $ळ т$ или одной из её собственных подгрупп: $1,2,3, \ldots ; m, 2 m, 3 m \ldots$ Общее свойство таких сред - отсутствие элементов симметрии, неизбежно устранивших бы пироэффект. Это центр симметрии (инверсии), поперечная к имеющейся оси плоскость симметрии, бесконечное множество косых и поперечных к имеющейся оси простых и зеркальных (или инверсионных, что не то же самое, но в данном контексте адекватно) осей симметрии. Совокупность всех потенциально возможных, но отсутствующих в среде элементов симметрии и есть её диссимметрия, «творящая явление», в данном случае - пироэффект.

Казалось бы, пример исчерпывает формулировку. Но встречаются разные употребления слов «объект, поле, явление». А.В. Шубников их различает: «Свойством симметрии могут обладать не только кристаллы и другие вещественные объекты, но и физические поля, а также и физические явления》 (с. 596). Терминологическая путаница возникает уже из-за того, что, следуя этимологии, 
можно считать объектами (т.е. объективно существующими) физические поля и явления (свойства, эффекты), а явлениями (т.е. тем, что явлено наблюдателю) - (видимые) объекты и (измеримые) физические поля. Нестрогое разделение понятий, их понимание из контекста - причина разночтений, порождающих дискуссии.

«Когда несколько различных явлений природы накладываются друг на друга, образуя одну систему, диссимметрии их складываются. В результате остаются лишь те элементы симметрии, которые являются общими для каждого явления, взятого отдельно» (с. 598). Это положение известно как принцип суперпозиции симметрий. Оно «является далеко не тривиальным распространением на физические явления тривиальной для геометрических фигур истины, заключающейся в том, что при соединении двух (или многих) не равных друг другу симметричных составляющих фигур в одну составную, в последней остаются лишь те элементы симметрии, которые являются общими для всех составляющчих фигур при заданном способе их размещуения в пространстве» (там же).

«В научных кругах большей известностью, нежели только что рассмотренный принцип суперпозиции симметрий, пользуются (без должного их понимания) три принципа симметрии П. Кюри, коими устанавливается связь между симметрией причины и следствия. Эту известность они получили, как нам кажется, благодаря тому, что в опубликованных М. Кюри воспоминаниях о муже они выделены курсивом и занумерованы как наиболее важные. Сам П. Кюри <..> курсивом их не выделяет и не нумерует. Вот эти принияипь:

1. Когда определённые причины порождают известные следствия, элементы симметрии причины должны содержаться в порождённых следствиях.

2. Когда известные следствия обнаруживают известную диссимметрию, эта последняя должна содержаться и в причинах, породивших эти следствия.

3. Положения, обратные двум предьлущуим, неправильны, по крайней мере, на практике, т.е. следствия могут быть симметричнее вызываюших их причин.

Трудность понимания этих принципов заключается в неясности, что в конкретных случаях следует понимать под “причиной” и “следствием” и что следует разуметь под их “симметрией”, и “диссимметрией”» (с. 599-600).

На наш взгляд, трудность понимания п.п. 1-3 этим не исчерпывается. Так, заключительная часть п. 3 явно следует из п. 1. При этом следствия будут симметричнее причин тогда и только тогда, когда элементы симметрии причин образуют собственную подгруппу в группе, образованной элементами симметрии следствий. Остаётся неясной оговорка «по крайней мере, на практике». Как будто на практике возможна ситуация, не предусмотренная корректной теорией. Далее, п. 2 логически не зависит от п. 1. Но вот вопрос: образуют ли в совокупности элементы симметрии и элементы диссимметрии (сами группу не образующие) причин ту же предельную группу симметрии Кюри (и всегда ли группу $/ \infty m$ покоящейся сферы?), что и элементы симметрии и элементы диссимметрии следствий? Вопрос требует специального рассмотрения путём перебора вариантов с учётом иерархии (схемы вложения) семи предельных групп симметрии Кюри.

Как бы то ни было, А.В. Шубников делает вывод, что «при применении принцииов П. Кюри можно и должно за симметрию свойств и явлений принимать симметрию тех величин <...> или тех фигур, коими они <.. > описываются» (с. 600-601), а «все три принциипа П. Кюри, связывающчие симметрию причины и симметрию следствия, в конечном счете, могут быть действительно сведеньл к им же сформулированному принциипу суперпозищии симметрий» (с. 602).

\section{Принцип Кюри в книге В.П. Афанасьева}

В книге В.П. Афанасьева (2018) изложена натурфилософская позиция, в основе которой - логический и онтологический подходы к познанию природы, дополняющие друг друга. «Bcлед за Лейбницем я разделил понимание симметрии на онтологический и логический уровни, что позволило дать ясную формулировку симметрии» (с. 7). Рассмотрение всей работы увело бы нас в сторону. Далее нас будет интересовать специальная глава «Принцип симметрии Кюри». Но прежде укажем на россыпь предваряющих её несообразностей. 
«Выяснилось, что отсутствует единое внятное определение симметрии, а как без него обсуждать симметрию?» (с. 7). «На мой взгляд, неудачи в попытках дать общее определение симметрии обусловлены невозможностью этой общей формулировки» (с. 60). Но чем плохо следующее определение: «Две фигуры называются взаимно равныли, если для каждой точки одной фигуры имеется соответственная точка другой фигуры, причём расстояние между любыми двумя точками одной фигуры равно расстоянию между двумя соответственными точками другой. Формулировка принадлежит немеикому геометру А.Ф. Мёбиусу (1790-1868)» (Попов, Шафрановский, 1964). Сегодня то же выражается короче: симметрия - отображение фигуры в себя или другую фигуру, сохраняющее метрику. Это определение лаконично и достаточно для построения геометрической кристаллографии. Что касается «единости», то многие математические понятия определяются разными, но эквивалентными способами.

«Интерпретаторы принципа симметрии свели вместе понятие диссимметрии в смысле Пастера и в смысле Кюри, что, на мой взгляд, недопустимо, поскольку под одним термином у них понимаются совершенно разные вещзи» (с. 7). Что действительно недопустимо, так это не читать по рассматриваемой проблеме А.В. Шубникова, который обсудил понятие диссимметрии в обоих смыслах. Невероятно, но в завершающем книгу списке литературы нет ни одной его работы. И.И. Шафрановский тоже никогда не путал диссимметрию по Пастеру и по Кюри. Тогда против каких интерпретаторов направлена критика?

«Возникает <..> необходимость метрики. <..> Метрика - порождение человеческого сознания, имеет универсальный характер, ею человек измеряет и объекты, и среду (пространство), определяет иные характеристики объектов и среды. Через систему мер человек характеризует мир» (с. 10). «Пространство - от-до, ближе-дальше, мера расстояния, точка отсчёта» (c. 16). Очевидно, автор не различает строго определяемые в математике категории метрики (расстояния) и меры, основополагающие в суждениях о пространстве. Множество с метрикой есть метрическое пространство, множество с мерой - пространство измеримое. Метрики могут задаваться через меры, но могут и без них. На всяком множестве можно задать метрику - в этом можно видеть её универсальность как базовой структуры мышления о протяжённости пространства. Но на одном множестве можно задать различные метрики - в этом их относительность, функциональность.

«Геометрия выросла из практических потребностей человека, из необходимости измерения объектов, измерений на местности. Но, постепенно абстрагировавшись <...>, геометрия получила самостоятельную жизнь. В результате от созериания тех или иных выделенных объектов можно легко перейти к абстрактным образам евклидовой геометрии, в том числе связанным с бесконечностью. То же математика в цчелом» (с. 12). Автор очень поверхностно представляет историю геометрии и математики в целом. Осмысление бесконечности (потенциальной, а тем более актуальной) происходило весьма трудно и долго. Создание Г. Кантором теории бесконечных множеств пошатнуло фундамент математики. А применительно к нашей теме, так ли легко было П. Кюри перечислить предельные группы симметрии, характеризующие все (но все ли?) известные состояния физического пространства?

«Зеркальная симметрия представляет собой закономерность, охватываюшую обе энантиоморфные модификации, и вне этих модификаций и их взаимоотномений не существует» (с. 31). Никоим образом! Зеркальная симметрия подразумевает энантиоморфные разновидности фигур, имеющих лишь элементы симметрии 1-го рода. Но она существует и для фигур, имеющих хотя бы одну плоскость в своей точечной группе симметрии.

«В онтологическом подходе симметрия рассматривается как сохранение реального объекта при трансляции во времени. <..> Причём это не обязательно чисто геометрическая симметрия» (с. 60). Заметим, что до сих пор речь шла именно о геометрической симметрии в её классическом варианте (без привлечения криволинейной симметрии, топологических инвариантов и т.д.). И если объект не имеет других признаков симметрии кроме сохранения во времени, то его следует считать скорее асимметричным. Что мы приобретём, если «в онтологическом подходе» призна- 
ем симметричным примитивный триклинный минерал лишь потому, что он стабилен в известном поле Р-Т-Х-параметров?

«А.В. Гадолин в 1867 г. вывел 32 точечные группы симметрии, в которых винтовые оси фигурируют в качестве полноправного элемента симметрии» (с. 72). Но всякая винтовая ось содержит трансляцию, не оставляющую на месте ни одной точки пространства и потому невозможную в точечной группе симметрии конечной фигуры. На самом деле А.В. Гадолин использовал в своём выводе и фактически ввёл в кристаллографию инверсионные оси симметрии (позднее в изложении предмета на первый план вышли зеркально-поворотные оси, сегодня - снова инверсионные), хотя и не дал им этого названия. Путать винтовые и инверсионные оси симметрии недопустимо даже в научно-популярном (тем более в научно-популярном) издании.

В наиболее интересной нам главе «Принцип симметрии Кюри» мы остановимся лишь на самых важных моментах. «"Симметрия порождающей среды как бы накладывается на симметрию тела, образующегося в этой среде. Получившаяся в результате форма тела сохраняет только те элементы собственной симметрии, которые совпадают с наложенными на него элементами симметрии среды" (Шафрановский, 1985). С этим положением нельзя не согласиться, но лишь отчасти, так как игнорируется активная роль объекта» (с. 68-69). С этим нельзя согласиться, так как И.И. Шафрановский вовсе не игнорирует «активную роль объекта». Фраза «элементы собственной симметрии» указывает именно на (физически обоснованную) претензию объекта обладать собственной симметрией. «B paзных местах статьи (имеется в виду статья П. Кюри - Ю.В.) проводится мысль, что диссимметрия - это отсутствие элементов симметрии» (с. 70). Отнюдь! П. Кюри говорит об отсутствии в среде лишь тех элементов симметрии, которые помешали бы состояться явлению. При этом в среде сохраняются те элементы симметрии, в совокупности образующие подгруппу группы его характеристической симметрии, которые явлению не мешают. Лишь в предельном случае эта подгруппа тривиальна, то есть элементы симметрии отсутствуют.

«И не понятно, каким образом отсутствие элементов симметрии у объекта или прочесса является причиной некоторого явления〉 (там же). Вновь обратимся к этимологии. Явление - не умозрительная идея, а то, что явлено «в онтологическом подходе», то есть реально. Здесь уместно вспомнить учение Д.П. Григорьева об онтогении (т.е. индивидуальном развитии) минералов. Оно целиком посвящено явлениям, то есть несовершенствам, по которым только и возможны реконструкции «биографий» минеральных индивидов. Именно в этом смысле реальные объекты и процессы, характеризуемые в категориях симметрии, становятся для нас явлениями лишь тогда, когда в них отсутствуют некоторые (допустимые в идеале) элементы симметрии. «В онтологическом подходе» идеал явлен быть не может.

«Количественно описать геометрическую симметрию через элементы симметрии невозможно, она или есть, или её нет» (с. 74-75). Очень даже возможно! Для этого служат порядки групп автоморфизмов, позволяющие говорить о меньшей или большей геометрической симметрии в числовом выражении.

«Касаясь исчезнувших элементов симметрии, И.И. Шафрановский вслед за П. Кюри называет их “диссимметрией” < ..>, “которая создаёт явление”, т.е. диссимметрия по определению динамична, тогда как симметрия статична, и необходимо понять суть диссимметрии и её взаимоотношение с симметрией» (с. 68-69). «Диссимметрия присуща не отдельно взятым объекту и среде, она помещена между ними <...>, и только в таком понимании диссимметрия может творить явление» (с. 75). Именно в этом - признании динамического характера диссимметрии и локализации её между объектом и средой - заключаются главные тезисы В.П. Афанасьева.

Хотя мы приблизились к концу обсуждения, приходится возвращаться к началам. Диссимметрия по Кюри - это всегда множество отсутствующих элементов симметрии. Вместе с присутствующими элементами симметрии (формально образующими группу) они образуют одну из семи (с учётом энантиоморфизма - десяти) предельных групп симметрии Кюри, характеризующих среды (физические пространства). В этой взаимной дополнительности и состоит взаимоотношение симметрии с диссимметрией. Ничего более! Симметрия и диссимметрия должны иметь один статус, что- 
бы их можно было объединять или противопоставлять. Динамичность последней может быть понята как геометрическая (впрочем, в той же мере как алгебраическая, теоретико-групповая) характеристика, но не как причина физического действия (импульса, силы).

В.П. Афанасьев прав в том, что о диссимметрии мы говорим лишь тогда, когда есть оппозиция двух сущностей, которые П. Кюри и А.В. Шубников называют причиной и следствием. Это может быть явление (пьезоэффект) в среде (кристалле турмалина) или физический объект (растущий / растворяющийся кристалл) в среде (потоке раствора). Но заметим, что лишь во втором случае есть пространственный зазор (он же контакт) двух сущностей, куда мы могли бы поместить диссимметрию, понятую как их физическое взаимодействие. В первом случае этот зазор имеет метафорический смысл. В итоге предлагаемая интерпретация диссимметрии не вписывается в её классическое геометрическое понимание и ничего не добавляет к физическому взаимодействию в его онтологическом рассмотрении.

\section{Принцип Кюри в статье Б.С. Левина}

Б.С. Левин (2018) рассматривает не только принцип Кюри, но и принцип ШубниковаШафрановского, понимая под таковым «возможности сосуществования в системе разносимметрийных признаков» (с. 136). Но в такой формулировке принципа ещё нет, недостаёт хотя бы вербальной формулы, связывающей две части суждения каким-либо логическим квантором. Далее два принципа объединены им в принцип Кюри-Шубникова-Шафрановского: «Симметрия процесса, влияющего на какие-то отдельные признаки (свойства, качества) системы, отражается в симметрийной характеристике этих признаков, формируя их по своему образу и подобию. Для нескольких прочессов: при разной симметрии процессов, влияющих на одну систему, каждый из них может формировать по своей симметрии какую-то часть (признаки) всей системы. В силу различия симметрий отдельных признаков общая симметрия всей системы при этом понижается (диссимметрии складываются)» (с. 143).

В объединённом принципе выделяются прямой и обратный аспекты. «Прямой аспект: признаки системы, различающиеся симметриями, сформированы разными процессами с аналогичными симметрийными характеристиками. Он напрямую приложим к разным геологическим ситуациям. Обратный аспект не столь однозначен: признаки системы с одинаковыми симметриями, вероятнее всего, созданы единым проиессом со сходной симметрийной характеристикой» (с. 136). Употребление слов «по своему образу и подобию», «аналогичными», «сходной» - шаг назад по сравнению с классической формулировкой принципа Кюри, в которой отношения (а именно - вложения друг в друга) элементов симметрии (и элементов диссимметрии) причины и следствия указаны строго.

Приведенные формулировки в значительной мере базируются на модельном примере. «Соберём модель гране- и объёмоцентрированной кубической решётки. В углах кубических ячеек закрепим железные шарики, в центрах граней-пластмассовые, в центрах ячеек-резиновые. Подвергнем модель действию трёх разнонаправленных потоков, совпадающих, для простоты, с осями декартовой системы координат. Пусть по оси абсиисс пройдет подкисленный водный поток. Железные шарики покроются ржавчиной, остальные не отреагируют. Ржавчина в силу её контролируемости потоком с симметрией конуса превратит шарики в эллипсоиды. Симметрия «железной» части системы понизится от кубической до тетрагональной с одной осью. Затем по оси ординат пустим поток горячего воздуха. Железным и резиновым шарикам ничего не будет, а пластмассовые оплавятся и впишутся в симметрию потока, понизив свою собственную симметрию до тетрагональной с осью, перпендикулярной «железной» оси. Наконеи, организуем вдоль оси аппликат поток воздуха, насыщенного песком и пылью. На него отреагируют только резиновые узль решётки, поглощающие твёрдые частицы ториевой стороной, тогда как пластмассовые и железные останутся неизменёнными. Получим ещё одну тетрагональную систему, перпендикулярную двум предьдущим. В итоге общая симметрия всей модели понизится от кубической до асимметричной (примитивной) - три разнонаправленные тетрагональные подсистемы модели аннулируют друг друга. 
Теперь обратим ситуацию: исследователь обнаруживает описанную систему в конечном состоянии. Её симметрия - примитивная. Как таковая она ничего не говорит ему о своей истории. Но если проанализировать раздельно три составные части системы, вылвив для каждой симметрию, то можно прийти к выводу о трёх разновременных формообразуюших потоках. Именно к такой догадке и может подтолкнуть исследователя принщип Кюри <... , устанавливающий связь между симметриями причины и следствия, иначе говоря, между симметриями процессов (воздействующих потоков) и их материальных производных (изменений в узлах решётки)» (с. 136-137).

«Модель подсказывает более глубокий подход к принципу Кюри - вычленение разных свойств объекта. Таким образом, полезная суть принщипа Кюри заключена не столько в его общей формулировке, сколько в указанных деталях, ранее не привлекавших к себе внимание» (с. 137). «Приниип Кюри следует дополнить положением о возможном наличии в системе признаков с различными симметриями» (с. 138).

Нам представляется, что автор модели запутал сам себя. Применительно к принципу Кюри важно знать, однородны ли последовательные воздействия на систему. В модели они разнородны - и с точки зрения разумного наблюдателя нет нужды искусственно создавать их суперпозицию ради последующего разложения на очевидные составные части. Да и сам автор замечает: «B не очень сложных геологических ситуациях геологи всегда видели изменения, последовательно накладывающиеся друг на друга» (с. 138). Иначе говоря, в модели явно различимы три самостоятельные ситуации, каждая из которых интерпретируема согласно принципу Кюри. Суть дела гораздо интереснее при наложении однородных, «прозрачных» друг для друга воздействий. Именно тогда система достигает компромисса, отвечающего пересечению групп симметрии, характеризующих каждое из воздействий.

Поясним сказанное, усложнив модель Б.С. Левина. Пусть каждый из потоков обладает дополнительным свойством - назовём его «цветом» - равно сообщаемым железным, пластмассовым и резиновым шарикам. Если потоки ортогональны трём базовым плоскостям кубической решётки, то к прежнему результату (трём тетрагональным подсистемам) добавится «цветная» с симметрией $3 m$. Должен ли наблюдатель теперь решить, что на систему повлияли четыре процесса? Очевидно, такой вывод был бы ошибочным. Пример показывает, что восстановление причин по следствиям при суперпозиции коррелированных (частично однородных) воздействий - нетривиальная задача. Но и здесь стоит подчеркнуть, что геологические процессы всегда некоммутативны (неперестановочны). Именно на этом основано чтение геологической летописи. Начиная с Н. Стенона, геология во всех разделах разработала для этого немалый арсенал методов, вначале - относительной, затем - абсолютной геохронологии.

\section{Заключение}

Отдадим должное В.П. Афанасьеву и Б.С. Левину - они пытались переосмыслить, уточнить, расширить и сделать более понятным принцип Кюри. По нашему мнению, на этот раз он устоял. Как теоретическую концепцию с высокой степенью абстракции и широким охватом явлений, его можно рассматривать изолированно, но применять на практике следует в совокупности с другими подходами. И всё же, что кроется за словами А.В. Шубникова (1956, с. 602): «Идеи П. Кюри в области учения о симметрии нельзя считать до кониа оформленными. Это сделают будущие поколения»?

Одно из направлений исследований сформулировано выше. Образуют ли в совокупности элементы симметрии и элементы диссимметрии причин ту же предельную группу симметрии Кюри (и всегда ли группу $\infty / \infty m$ покоящейся сферы?), что и элементы симметрии и элементы диссимметрии следствий? Перебор вариантов с учётом иерархии предельных групп симметрии Кюри и поиск их природных прототипов - возможный способ решения.

По ходу изложения был задан вопрос: все ли состояния физического пространства предусмотрел П. Кюри в перечне предельных групп симметрии? Простейшей из них, вкладываемой во все другие, является группа $\infty$ вращающегося конуса. Но она не обладает той степенью примитивности, какой обладает подгруппа 1 любой алгебраической группы. Вспомним о живом веществе, как 
его представлял В.И. Вернадский (1987). Его суть - непрерывное изменение в каждой точке, согласованное в неоднородной системе живого организма более сложно, чем для однородных физических полей. Как среда оно не характеризуется ни одной из предельных групп симметрии Кюри. По-видимому, только живое вещество, не допускающее самосовмещения даже в мгновенном мысленном акте, может отвечать примитивной группе 1. Это вполне подтверждается крайней искажённостью биоминеральных индивидов и агрегатов. При этом, не обладая осью $\infty$, примитивная группа 1 не является в строгом смысле предельной группой Кюри.

Принцип Кюри сформулирован в рамках классической симметрии. Возможны ли его расширения за счёт групп гомологии (Михеев, 1961), антисимметрии (Шубников, 1951 а, 1951 б, 1966; Копцик, 1966), цветной (Белов, Тархова, 1956; Дубов и др., 1985), криволинейной симметрии (Наливкин, 1925, 1951; Падуров, 1926; Дубов, 1971) и ещё более общих преобразований, сохраняющих топологические инварианты? На этом пути, следуя логике П. Кюри, нужно всякий раз определять смысл перехода к предельным группам и находить их полный перечень, оставаясь в рамках реальности.

\section{Литература}

1. Афанасьев В.П. Человек и природа. Новосибирск: Изд-во СО РАН, 2018. 93 с.

2. Белов Н.В., Тархова Т.Н. Группы цветной симметрии // Кристаллография. 1956. Т. 1. № 1. С. 4-17.

3. Вернадский В.И. Химическое строение биосферы Земли и её окружения. М.: Наука. 1987. 340 c.

4. Войтеховский Ю.Л. Принцип Кюри и гранаты г. Макзапахк // Докл. АН. 2005. Т. 400. № 3. С. 355-358.

5. Войтеховский Ю.Л., Степенщиков Д.Г. Реальные ромбододекаэдры: теория и приложения к гранатам г. Макзапахк, Зап. Кейвы, Кольский п-ов // Зап. РМО. 2005. № 1. С. 97-103.

6. Дубов П.Л. Криволинейная симметрия. Автореф. дис. на соискание уч. ст. канд. геол.-мин. наук. Л.: Издво ЛГИ. 1971. $11 \mathrm{c.}$

7. Дубов П.Л., Франк-Каменецкий В.А., Шафрановский И.И. Обобщённая симметрия: учебное пособие. Л.: Изд-во ЛГУ. 1985. 76 с.

8. Копцик В.А. Шубниковские группы. М.: Изд-во МГУ. 1966. 723 с.

9. Кюри М. Пьер Кюри. Л.: Научн. хим.-тех. изд-во. 1924. 78 с.

10. Кюри П. О симметрии в физических явлениях: симметрия электрического и магнитного полей // Избр. труды. М.-Л.: Наука. 1966. С. 95-113.

11. Левин Б.С. Определение понятия «минерал» // Региональная геология, минералогия и полезные ископаемые Кольского п-ова. Тр. ХІІІ Всерос. Ферсмановской научн. сессии. Апатиты, 4-5 апр. 2016 г. Апатиты: Изд-во К \& М, 2016 а. С. 40-44.

12. Левин Б.С. Определение понятия «горная порода» // Региональная геология, минералогия и полезные ископаемые Кольского п-ова. Тр. ХІІІ Всерос. Ферсмановской научн. сессии. Апатиты, 4-5 апр. 2016 г. Апатиты: Изд-во К \& М. 2016 б. С. 44-48.

13. Левин Б.С. Определение понятия «формация» // Региональная геология, минералогия и полезные ископаемые Кольского п-ова. Тр. XIII Всерос. Ферсмановской научн. сессии. Апатиты, 4-5 апр. 2016 г. Апатиты: Изд-во К \& М, 2016 в. С. 49-53.

14. Левин Б.С. Систематика геологических наук // Региональная геология, минералогия и полезные ископаемые Кольского п-ова. Тр. ХІІІ Всерос. Ферсмановской научн. сессии. Апатиты, 4-5 апр. 2016 г. Апатиты: Изд-во К \& М, 2016 г. С. 54-56.

15. Левин Б.С. Приложения принципа Кюри в геологии // Зап. РМО. 2018. № 6. С. 136-144.

16. Михеев В.И. Гомология кристаллов. Л.: Гостоптехиздат. 1961. 208 с.

17. Наливкин Д.В. Элементы симметрии органического мира // Изв. Биол. НИИ при Пермском ун-те. 1925. Т. 3. Вып. 8. С. 291-297.

18. Наливкин Д.В. Криволинейная симметрия // Кристаллография. Тр. Фёдоровской научной сессии 1949 г. М.: Металлургиздат. 1951. С. 15-23.

19. Падуров Н.Н. О криволинейной симметрии // Зап. РМО. 1926. № 2. С. 314-334.

20. Пастер Л. Исследования о брожениях. М.-Л.: Сельхозгиз. 1937. 488 с.

21. Пастер Л. Избр. труды. М.: Изд-во АН СССР, 1960. Т. 1 - 1012 с. Т. 2 - 836 с.

22. Попов Г.М., Шафрановский И.И. Кристаллография. М.: Высшая школа, 1964. 370 с.

23. Шафрановский И.И. Внешняя симметрия реальных кристаллов и симметрия питающей среды // Зап. ВMO. 1954. № 3. С. 198-211.

24. Шафрановский И.И. Группы симметрии в структурной петрологии // Зап. ВМО. 1956. № 4. С. 491-497.

25. Шафрановский И.И. Пьер Кюри - кристаллограф // Тр. ИИЕТ АН СССР. 1957. Т. 19. С. 84-94.

26. Шафрановский И.И. Ложные формы кристаллов и их минерагенетическое значение // Зап. ВМО. 1959. № 1. С. 13-20. 
27. Шафрановский И.И. К вопросу об уточнении универсального принципа симметрии Кюри // Зап. ВМО. 1964. № 4. С. 460-463.

28. Шафрановский И.И. Несколько слов по поводу русского перевода трудов П. Кюри // Зап. ВМО. 1966. № 6. С. 758-760.

29. Шафрановский И.И. Лекции по кристалломорфологии. М.: Высшая школа. 1968 а. 174 с.

30. Шафрановский И.И. Симметрия в природе. Л.: Недра. 1968 б. 184 с. (Изд. 2-е, 1985).

31. Шафрановский И.И. Моделирование внешней диссимметрии кристаллической фор-мы // Кристаллография. 1971 а. № 6. С. 1097-1100.

32. Шафрановский И.И. Принцип Кюри и динамическая симметрия // Симметрия в природе Тез. докл. совещ. 25-29 мая 1971 г. Л.: Обл. правл. науч.-тех. горн. об-ва. 1971 б. С. 7-11.

33. Шафрановский И.И. Кристаллические формы как индикаторы особенностей минералообразующей среды // Типоморфизм минералов и его практическое значение. М.: Недра. 1972. С. 64-67.

34. Шафрановский И.И. Динамическая симметрия в кристаллографии, минералогии, петрографии в органическом мире // Зап. ЛГИ. 1974 а. Т. 67. № 2. С. 174-184.

35. Шафрановский И.И. Универсальный принцип симметрии-диссимметрии в минералогической кристаллографии // Минералогенезис. Изв. Геол. ин-та. Сер. геохим., минерал. и петрогр. Т. 23. София, 1974 б. С. $63-68$.

36. Шафрановский И.И. Форма минералов - индикатор симметрии внешней среды // Тр. ИГ Кар ФАН СССР. Вып. 27. 1975. С. 4-9.

37. Шафрановский И.И., Корень Р.В., Глазов А.И. Определение ориентировки природного кристалла относительно элементов симметрии питающей среды // Зап. ВМО. 1972. № 3. С. 372-374.

38. Шафрановский И.И., Плотников Л.М. Симметрия в геологии. Л.: Недра. 1975. 144 с.

39. Шубников А.В. Диссимметрия // Вопросы минералогии, геохимии и петрографии. М.-Л.: Изд-во АН СССР. 1946. С. 128-163.

40. Шубников А.В. Симметрия и антисимметрия конечных фигур. М.: Изд-во АН СССР, 1951 а. 172 с.

41. Шубников А.В. Перспективы развития учения о симметрии // Тр. Фёдоровской научной сессии 1949 г. М.: Металлургиздат. 1951 б. С. 33-47.

42. Шубников А.В. О работах Пьера Кюри в области симметрии // Успехи физ. наук. 1956. Т. 59. Вып. 4. C. 591-602.

43. Шубников А.В. Проблема диссимметрии материальных объектов. М.: Изд-во АН СССР. 1961.56 с.

44. Шубников А.В. Антисимметрия. Докл. на 7-ой Генеральной ассамблее, межд. конгрессе и симпозиуме. М.: Наука. 1966. 28 c.

45. Шубников А.В. У истоков кристаллографии. М.: Наука. 1972. 52 с.

46. Amstutz G.C. La symétrie comme critère génétique en géochimie et en gîtologie// Schweiz. mineral. und petrogr. Mitt. 1966. Bd 46. N 2. S. 329-335.

47. Curie P. Sur la symetrie dans les phenomenes physiques, symetrie d'un champ electrique et d'un champ magnetique // J. de Physique. 1894. V. 3. N 3. P. 393-416.

48. Curie P. Sur la symetrie dans les phenomenes physiques, symetrie d'un champ electrique et d'un champ magnetique // Oeuvres de P. Curie. Paris. 1908. P. 118-141.

49. Kirchmayer M. Das Symmetrie-Konzept von Curie 1884 in der Makrogefügekunde // N. Jb. Geol. Paläont. Abh. 1965. Bd 122. N 3. S. 343-350.

50. Kirchmayer M. Die Beschreibung tektonischer Stockwerke mit Hilfe des Symmetrie-Konzept // Geol. Föreningens i Stockholm Förhandlingar. Stockholm, 1966. Bd. 88. S. 75-89.

51. Kirchmayer M. Beispiele zum Curie'schen Symmetrie-Konzept, entnommen aus der Kluft und Schlechtentektonik des Ruhrgebietes und aus der Sedimentologie. Sonderdrück aus den Mitteilungen der Geologischen Gesellschaft Essen. H. 6. Essen: Schrepper \& Co, 1968. 15 S.

52. Pasteur L. Nouvelles recherches sur les relations qui peuvent exister entre la forme cristalline, la composition chimique et le phénomène rotatoire moléculaire // Ann. chimie et de phys. 1853. 38(3). 437.

53. Pasteur L. Recherches sur la dissimétrie moléculaire des produits organiques naturels. Paris. 1861.

54. Paterson M.S., Weiss L.E. Symmetry concepts in the structural analysis of deformed rocks // Geol. Soc. Amer. Bull. 1961. V. 72. P. 841-882.

55. Sander B. Gefügekunde der Gesteine. Wien: Springer, 1930. 352 S.

56. Sander B. Einführung in die Gefügekunde der geologischen Korper. Wien: Springer. Bd. 1. 1948. 215 S. Bd. 2. 1950. $409 \mathrm{~S}$

57. Turner F.J., Weiss L.E. Structural analysis of metamorphic tectonites. New York: McGraw-Hill, 1963. 545 p.

58. Voytekhovsky Y.L. The Curie principle and garnets from Mt. Makzapakhk // Dokl. Earth Sci. 2005. V. 400. N 1. P. 22-24.

59. Voytekhovsky Y.L., Stepenshchikov D.G. On the real crystal rhombododecahedra // Acta Cryst. 2004. A60. P. 582-584. 